\title{
A FINITE CAPACITY ANALOGUE OF THE KOEBE ONE-QUARTER THEOREM
}

\author{
ROBIN CUNNINGHAM
}

(Communicated by Albert Baernstein)

\begin{abstract}
A variational method is used to determine the largest disk about the origin covered by the image of every normalized univalent function that maps the unit disk onto a region of prescribed logarithmic capacity (transfinite diameter).
\end{abstract}

\section{INTRODUCTION}

Let $S$ be the usual class of functions $f(z)=z+a_{2} z^{2}+\cdots$ that are analytic and univalent in the unit disk D. In 1916 Bieberbach [1] established the following theorem.

Theorem A (Koebe one-quarter theorem). The range of every function $f \in S$ contains the disk $\left\{w:|w|<\frac{1}{4}\right\}$.

Koebe [9] had earlier proven that the theorem is true for some absolute radius $\rho>0$. The Koebe function

$$
k(z)=\frac{z}{(1-z)^{2}}=z+2 z^{2}+3 z^{3}+\cdots
$$

and its rotations show that the radius $\frac{1}{4}$ is best possible.

For $R>1$, we define $S_{R}$ to be the set of functions $f \in S$ whose image $f(\mathbf{D})$ has logarithmic capacity (transfinite diameter) equal to $R$. Recently some attention has been focused on extremal problems in the class $S_{R}$. Duren and Schiffer [5] found the maximum modulus of the second coefficient $a_{2}$ among functions $f \in S_{R}$. The author [3] later established sharp upper bounds for $|f(\zeta)|$ where $\zeta$ is a fixed point in $\mathbf{D}$ and $f$ ranges over the class $S_{R}$. In this paper we consider the minimum modulus problem and establish the following analogue of the Koebe one-quarter theorem:

Received by the editors March 13,1992; presented to the AMS at their annual meeting in January, 1991.

1991 Mathematics Subject Classification. Primary 30C25, 30C45, 30C85, 33A25.

Key words and phrases. Univalent functions, maximal covered disks, extremal problems, variational methods, quadratic differentials, logarithmic capacity, transfinite diameter, elliptic integrals.

This paper constitutes a portion of the author's Ph.D. thesis at the University of Michigan under the supervision of Professor Peter Duren. 
Theorem 1. For $R>1$, every function $f \in S_{R}$ maps $\mathbf{D}$ onto a region containing the open disk about the origin with radius

$$
r=\frac{A-1}{4 A}\left(\frac{\sqrt{A}+1}{\sqrt{A}-1}\right)^{1 / \sqrt{A}},
$$

where $A$ is the unique positive value satisfying the relation

$$
R=\frac{(A-1)^{2}}{16 A}\left(\frac{\sqrt{A}+1}{\sqrt{A}-1}\right)^{1 / \sqrt{A}+\sqrt{A}} .
$$

For each choice of $R>1, r_{0}$ is the maximal such radius.

The proof relies on a variational method used to solve a particular extremal problem in the class $S_{R}$. For general information regarding transfinite diameter and logarithmic capacity, see [6, Chapter VII] or [8, Chapter 16].

\section{A MINIMUM MODULUS PROBLEM}

We begin by addressing the extremal problem to find $\min _{g \in S_{R}}|g(\zeta)|$ for a given $R>1$ and $0<\zeta<1$. Using the variational methods in [3] or [5] we see that $S_{R}$ contains an extremal solution $f$ to this problem and that the boundary of $f(\mathbf{D})$ consists of analytic arcs lying on trajectories of the quadratic differential

$$
Q(w) d w^{2}=\frac{(b+1) B-w}{(w-B) w^{2}} d w^{2}>0, \quad B=f(\zeta)>0 .
$$

Details for the determination of (1) are similar to those in [3] and will be suppressed here. In this quadratic differential, the parameter $b$ is positive and is determined by $R$ and $\zeta$. The quadratic differential (1) has a simple zero at $w=(b+1) B$, a simple pole at $w=B$, and double poles at $w=\infty$ and $w=0$. It is easy to show that the trajectories of (1) are symmetric in the real axis and that $B>0$. We are thus able to sketch the trajectory structure of (1) in Figure 1. (See [10, Chapter 8] for an overview of trajectory structures of quadratic differentials.) In Figure 1, only those trajectories that surround both $w=0$ and $w=B$ may contain arcs, which form the boundary of $f(\mathbf{D})$.

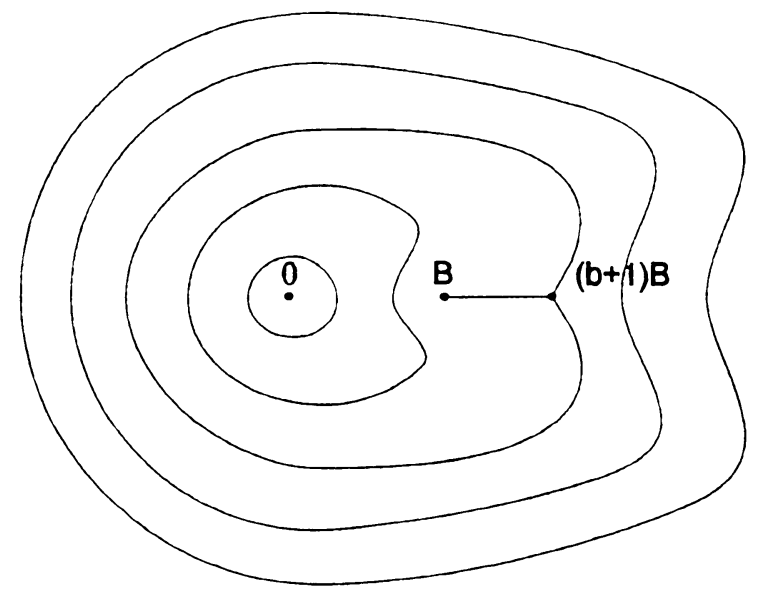

FiguRE 1. Trajectories of the quadratic differential (1). 
We define $\Gamma$ to be the boundary of $f(\mathbf{D})$ and parameterize $\Gamma$ by $w=f\left(e^{i t}\right)$. Using (1) we then obtain

$$
F(z)=\frac{z^{2} f^{\prime}(z)^{2}}{f(z)^{2}} \frac{[(b+1) B-f(z)]}{B-f(z)} \geq 0, \quad|z|=1 .
$$

Since $f \in S$, it is clear that $F$ is analytic in D except for a removable singularity at $z=0$ and a simple pole at $z=\zeta$. In order to arrive at a convenient expression for $F(z)$, one shows that $F(z)$ is analytic and nonvanishing on $|z|=1$ except for at most one double zero when $\Gamma$ has an internal spire or a corner. (The proof for this follows just as in $[5, \S 3]$.) Then by the Schwarz reflection principle, $F(z)$ has a meromorphic extension to the Riemann sphere satisfying $F(1 / \bar{z})=\overline{F(z)}$; therefore, $F(z)$ has the form

$$
F(z)=\frac{A\left(z-z_{0}\right)\left(1-z z_{0}\right)}{(z-\zeta)(1-\zeta z)},
$$

where $f\left(z_{0}\right)=(b+1) B$ when $w=(b+1) B$ lies in $f(\mathbf{D})$. Thus it is immediate from (3) that $z_{0}>0$ and that $A$ is real. It happens that the point $w=(b+1) B$ is in $\overline{F(\mathbf{D})}$ for each of the trajectories of (1) which enclose both $w=0$ and $w=B$.

By considering the trajectory structure of $(3)$ (see $[5, \S 3]$ ), we see that our extremal function $f$ maps the unit disk onto a region whose boundary $\Gamma$ falls into one of the following two cases:

(i) $\Gamma$ has as its outer boundary a Jordan curve containing the point $w=$ $(b+1) B$ and has a corner there. Also $\Gamma$ contains a horizontal slit $[\eta,(b+1) B]$, which may have length zero. In any event $\eta>B$, or else $f(\mathbf{D})$ would not contain $B$.

(ii) $\Gamma$ is an analytic Jordan curve and the points $w=0, w=B$, and $w=(b+1) B$ all lie in $f(\mathbf{D})$.

We will handle case (i) first. It will turn out that, for a fixed $R$ and for all $\zeta$ sufficiently close to 1 , the extremal function $f$ maps $\mathbf{D}$ onto a region of the type described in case (i). Thus case (i) is the more important in the proof of Theorem 1, since it is concerned with the behavior of solutions of the minimum modulus problem as $\zeta$ approaches 1 .

\section{CORNER WITH RADIAL SLIT}

In case (i), $F(z)$ as given by (2) and (3) has a double zero on the unit circle and $z_{0}>0$ so that

$$
F(z)=\frac{-A(1-z)^{2}}{(z-\zeta)(1-\zeta z)} .
$$

Now upon considering (4) along with (2) we have three parameters $A, B$, and $b$. To express them in terms of $R$ and $\zeta$ we need three relations among the variables.

For a first relation we evaluate $\lim _{z \rightarrow 0} F(z)$ in (2) and (4) to see that

$$
(b+1)=A / \zeta \text {. }
$$

It will be useful to note that this implies $A>0$. 
For a second relation we make use of the analytic completion of Green's function for the complement of $\overline{f(\mathbf{D})}$ (with singularity at infinity);

$$
G(w)=\int_{(b+1) B}^{w} \sqrt{\frac{w-(b+1) B}{w-B}} \frac{d w}{w} .
$$

Using $[7, \S 2.26]$ we obtain

$$
\operatorname{Re}\{G(w)\}=\log |w|+\log \left|\frac{4}{b B}\right|-\sqrt{\frac{A}{\zeta}} \log \frac{\sqrt{A / \zeta}+1}{\sqrt{A / \zeta}-1}+O\left(\frac{1}{w}\right),
$$

but since $\operatorname{Re}\{G(w)\}$ is Green's function, we have

$$
\operatorname{Re}\{G(w)\}=g(w)=\log |w|-\log R+O(1 / w) .
$$

Now by comparing constant terms we see that

$$
\log R=\log \frac{b B}{4}+\sqrt{\frac{A}{\zeta}} \log \frac{\sqrt{\frac{A}{\zeta}}+1}{\sqrt{\frac{A}{\zeta}}-1} .
$$

For a final third relation we use the fact that $f^{\prime}(0)=1$ along with equality of the integrals

$$
\int_{f(\varepsilon)}^{B} \sqrt{\frac{(b+1) B-w}{B-w}} \frac{d w}{w}=\sqrt{A} \int_{\varepsilon}^{\zeta} \frac{1-z}{\sqrt{(\zeta-z)(1-\zeta z)}} \frac{d z}{z},
$$

where (2) and (4) have been used. Upon calculation the right integral is determined to be

$$
\sqrt{\frac{A}{\zeta}} \log \frac{4 \zeta}{(1+\zeta)^{2}}-\sqrt{\frac{A}{\zeta}} \log \varepsilon+O(\varepsilon) .
$$

The left integral is calculated to be

$$
-\sqrt{b+1} \log f(\varepsilon)+\sqrt{b+1} \log \frac{4(b+1) B}{b}+\log \frac{\sqrt{b+1}-1}{\sqrt{b+1}+1}+O(\varepsilon) .
$$

Setting (9) equal to (8) and letting $\varepsilon$ approach zero, we use the fact that

$$
\lim _{\varepsilon \rightarrow 0}\left[\log \frac{f(\varepsilon)}{\varepsilon}\right]=0
$$

to obtain the relation

$$
\log B=\log \frac{\zeta}{(1+\zeta)^{2}}+\log \frac{A / \zeta-1}{A / \zeta}+\sqrt{\frac{\zeta}{A}} \log \frac{\sqrt{\frac{A}{\zeta}}+1}{\sqrt{\frac{A}{\zeta}}-1}
$$

where (5) has been used.

Before continuing further we pause to verify that $(10)$ gives a sharper estimate for $|f(\zeta)|$ than does Bieberbach's estimate [1], namely,

$$
|f(\zeta)| \geq \frac{|\zeta|}{(1+|\zeta|)^{2}}
$$

for the full class $S$. To see that (10) improves on (11) for the class $S_{R}$ we need to show that the sum of the last two terms in (10) is positive. First note that 
as $A$ approaches infinity, this sum tends to zero. Taking a derivative reveals that $\partial B / \partial A<0$ for $A>\zeta$, so the sum in question is positive for $\zeta<A<\infty$ (which corresponds to $b>0$ ). Thus (10) is an improvement on Bieberbach's estimate (11) for each $R$ and $\zeta$, whatever the value of $A$.

Next we use (6) to eliminate $B$ from (10) to obtain

$$
\begin{aligned}
\log R= & \log \frac{\zeta}{(1+\zeta)^{2}}-\log 4 \\
& +\log \left(\sqrt{\frac{A}{\zeta}}-2+\frac{\zeta}{A}\right)+\left(\sqrt{\frac{\zeta}{A}}+\sqrt{\frac{A}{\zeta}}\right) \log \frac{\sqrt{\frac{A}{\zeta}}+1}{\sqrt{\frac{A}{\zeta}}-1} .
\end{aligned}
$$

In order to assert that (12) implicitly determines $A$ in terms of $R$ and $\zeta$ we show that for each fixed $\zeta, \partial R / \partial A>0$.

We can modify the lemma $[3, \S 6]$ to show that the derivative of $B$ with respect to $R$ is negative for a fixed value of $\zeta$. Since we saw above that $B$ is monotone with respect to the parameter $A$, we may conclude immediately that $R$ is monotone with respect to $A$. This proves that $A$ is implicity determined in terms of $R$ and $\zeta$ by (12). Thus when case (i) occurs, $B$ is given parameterically in terms of $R$ and $\zeta$ by (10) and (12).

Since $R$ is monotone with respect to $A$, at most one extremal function may map the unit disk onto regions of the type found in case (i).

\section{ANALYTIC JoRdAN CURVES}

For case (ii) we again take $F(z)$ to be defined by (2) and (3). Since the point $w=(b+1) B$ lies in $f(\mathbf{D})$, we know that $f\left(z_{0}\right)=(b+1) B$. This implies that $\zeta<z_{0}<1$. In (2) and (3) we have four parameters: $A, B, b, z_{0}$. For the proof of Theorem 1, we shall require only one relation among these parameters.

If the extremal function $f$ falls into case (ii), let $w_{1}=f(1)$ be the point where $\Gamma$ meets the positive real axis and consider

$$
H(w)=\int_{w_{1}}^{w} \sqrt{\frac{w-(b+1) B}{w-B}} \frac{d w}{w}=G(w)-G\left(w_{1}\right),
$$

where $G(w)$ is as defined in $\S 2$. Since $\Gamma$ consists of arcs lying in trajectories of (1), we see that $H(w)$ is the analytic completion of Green's function for the complement of $\overline{f(\mathbf{D})}$. Therefore

$$
\operatorname{Re}\{H(w)\}=g(w)=\log |w|-\log R+O(1 / w) .
$$

Now upon considering (6) and the analysis leading up to it, we see that

$$
\log R=\log \frac{b B}{4}+\sqrt{b+1} \log \frac{\sqrt{b+1}+1}{\sqrt{b+1}-1}+\operatorname{Re}\left\{G\left(w_{1}\right)\right\}
$$

Using this relation we will show in the next section that any functions that fall into case (ii) map the unit disk onto a region containing a disk with radius $r>r_{0}$ where $r_{0}$ is defined in Theorem 1 . 


\section{Maximal COVERed DisKS}

As indicated earlier our results regarding maximal covered disks will rely on the fact that, for every $R>1$, we may choose $\zeta_{R}$ so that for $\zeta_{R}<\zeta<1$ the extremal function for $R$ and $\zeta$ maps $\mathbf{D}$ onto a region of the type described in $\S 2$. This fact is proven via a contradiction argument.

First, fix $R>1$. Now suppose that for this $R$ there exist values of $\zeta$ arbitrarily close to 1 so that the extremal function $f_{R, \zeta}$ maps $\mathbf{D}$ onto a region bounded by an analytic Jordan curve. Then as we choose such $\zeta$ close to 1 the point $w=(b+1) B$ remains trapped between $w=B$ and $w_{1}=f(1) \leq 4 R$. (Since $f(\mathbf{D})$ has logarithmic capacity $R, f(\mathbf{D})$ must lie entirely within the disk $w<4 R$. See [4, Chapter 10].) Thus, for values of $\zeta$ approaching 1 ,

$$
G\left(w_{1}\right)=\int_{(b+1) B}^{w_{1}} \sqrt{\frac{w-(b+1) B}{w-B}} \frac{d w}{w}
$$

approaches 0 . Similarly, for $\zeta$ approaching 1 in case (ii), $b$ approaches 0 . We define $B_{\zeta}=\min _{f \in S_{r}}|f(\zeta)|$. Now by taking the limit as $\zeta$ approaches 1 in equation (15), we see after a calculation that

$$
\log R=\lim _{\zeta \rightarrow 1}(\log R)=\lim _{\zeta \rightarrow 1}\left(\log B_{\zeta}\right) ;
$$

but $R>1$ while $B_{\zeta} \leq 1$ for all $\zeta(0<\zeta<1)$, thus we have a contradiction.

Thus for every $R>1$, we may assume for $\zeta$ sufficiently close to 1 that $B_{\zeta}$ is given by (10) in which $A$ is determined by (12). Now by letting $\zeta$ approach 1 in (10) and (12), we obtain Theorem 1. Table 1 provides some sample values of the maximal radius of the covered disks for a few choices of transfinite diameter $R$. For $R=1, r_{0}$ is given by the identity function while the Koebe function provides the extremal value for $R=\infty$.

TABLE 1

\begin{tabular}{|r|c|}
\hline \multicolumn{1}{|c|}{$R$} & $r_{0}$ \\
\hline 1.0 & 1.0000 \\
1.001 & 0.9228 \\
1.01 & 0.7991 \\
1.1 & 0.5650 \\
2.0 & 0.3357 \\
10 & 0.2623 \\
100 & 0.2512 \\
1000 & 0.2501 \\
$\infty$ & 0.2500 \\
\hline
\end{tabular}




\section{REFERENCES}

1. L. Bieberbach, Über die Koeffizienten derjenigen Potenzreihen, welche eine schlichte Abbildung des Einheitskreises vermitteln, S. B. Preuss. Akad. Wiss. 35 (1916), 940-955.

2. P. F. Byrd and M. D. Friedman, Handbook of elliptic integrals of engineers and physicists, Springer-Verlag, Berlin, Göttingen, and Heidelberg, 1954; 2nd edition, 1971.

3. R. Cunningham, Univalent functions of given transfinite diameter: a maximum modulus problem, Ann. Acad. Sci. Fenn. (to appear).

4. P. L. Duren, Univalent functions, Springer-Verlag, Heidelberg and New York, 1983.

5. P. L. Duren and M. Schiffer, Univalent functions which map onto regions of given transfinite diameter, Trans. Amer. Math. Soc. 323 (1991), 413-428.

6. G. M. Goluzin, Geometric theory of functions of a complex variable, Gosudarstv. Izdat. Tehn.-Teor. Lit., Moscow-Leningrad, 1952; German transl., Deutscher Verlag, Berlin, 1957; 2nd edition, Izdat. "Nauka”, Moscow, 1966; English transl., Amer. Math. Soc., Providence, RI, 1969.

7. I. Gradsteyn and I. Ryzhik, Table of integrals, series and products, Academic Press, New York, 1965; enlarged ed., 1980.

8. E. Hille, Analytic function theory, Vol. II, Ginn and Co., Boston, MA, 1962; second ed., Chelsea, New York, 1987.

9. P. Koebe, Über die Uniformisierung beliebiger analytischer Kurven, Nachr. Akad. Wiss. Göttingen Math.-Phys. Kl. (1907), 191-210.

10. Ch. Pommerenke, Univalent functions, Vandenhoeck and Ruprecht, Göttingen, 1975.

Department of Mathematics, Trenton State College, Trenton, New Jersey 08650

E-mail address: RobinC@Trenton.Edu 